\title{
HIV risk behaviours among injecting drug users in Vietnam: A review
}

\author{
Tanvir Ahmed ${ }^{1, ~ *, ~ N g u y e n ~ T h a n h ~ L o n g ², ~ P h a n ~ T h i ~ T h u ~ H u o n g ~}{ }^{2}$, Chiao Tzu Patricia Lee ${ }^{3}$, \\ Donald Edwin Stewart ${ }^{1}$
}

${ }^{1}$ Griffith University, School of Medicine, South Bank Campus, 226 Grey Street, Brisbane, Queensland, Australia

${ }^{2}$ Vietnam Authority of HIV/AIDS Control, Lane 135/3 Nui Truc Street, Ba Đinh District, Hanoi, Vietnam

${ }^{3}$ Griffith University, School of Medicine, Gold Coast Campus, Parklands Drive, Southport, Queensland, Australia

\section{Email address:}

tanvir.ahmed@griffithuni.edu.au (T. Ahmed), longvaac@gmail.com (N. T. Long), huongphanmoh@gmail.com (P. T. T. Huong), patricia.lee@griffith.edu.au (C. T. P. Lee), donald.stewart@griffith.edu.au (D. E. Stewart)

\section{To cite this article:}

Tanvir Ahmed, Nguyen Thanh Long, Phan Thi Thu Huong, Chiao Tzu Patricia Lee, Donald Edwin Stewart. HIV Risk Behaviours among Injecting Drug Users in Vietnam: A Review. Science Journal of Public Health. Vol. 2, No. 3, 2014, pp. 209-215.

doi: $10.11648 /$ j.sjph.20140203.22

\begin{abstract}
Background: There is high prevalence of HIV among Injecting Drug Users (IDUs) in Vietnam with a national level prevalence just under 20\%. Both drug and sex related risk behaviors are widespread among IDUs. The objective of this review is to analyze HIV risk behaviors and identify the epidemiologic implications. Methods: Major databases were searched during November-December, 2011. Finally, 55 peer-reviewed articles were identified and reviewed to focus on risk behaviors of IDUs, Female Sex Workers (FSW) and drug policy and harm reduction program related issues. Results: Young injectors are involved in risk behaviors that include frequent sexual connections with FSWs. Our review found that young IDU subgroups ( $\leq 30$ years) are engaged in high risk behaviors and have limited access to harm reduction services. Sexual risk factors associated with drug injecting FSWs may become the catalyst for a future heterosexual transmission if the prevalence rises further. Future research should focus on young injectors to investigate the structure and characteristics of networks to help guide harm reduction programs, including IDU subgroups. Conclusion: Vietnam has still a window of opportunity to learn from the experience of neighboring countries and, after modification, to incorporate preventative services suited to the needs of young injectors, into existing programs.
\end{abstract}

Keywords: HIV, AIDS, Vietnam, Injecting Drug User, Risk Behaviors

\section{Introduction}

There is a high prevalence of HIV among Injecting Drug Users (IDUs) in Vietnam, with a national level prevalence, according to Integrated Biological and Behavioral Surveillance (IBBS) survey in 2009, of just under 20\% [1]. IDUs have remained as the predominant risk group driving the HIV epidemic from its start [1-3].

Since HIV emerged in Vietnam in 1990, the epidemic has been characterized by sub-epidemics, primarily among IDUs, in most of the provinces [4, 5]. The explosive spread of HIV infection among IDUs was first recognized in 1993 especially in southern Vietnam [6]. In that year alone 945 cases were reported, of which $87 \%$ sero-positive persons were IDUs [7]. During the period of peak HIV prevalence among IDUs, a highly variable rate across regions ranging between zero and $89.4 \%$ was documented [8]. Also, the prevalence rate has been found to vary amongst IDUs in community settings or treatment centres [9], subgroups such as older [10], younger and new users [11-13]. There is also a high prevalence among young ethnic IDUs in the border provinces (south China and north Vietnam) [14]. Although, the surveillance data show a slight reduction in overall HIV prevalence among IDUs from 20.27\% (2008) to $18.44 \%$ (2009), variation exists across provinces [1]. Recent IBBS data [15] along with other research [11, 13, 16] show variability across the country with high HIV prevalence in some provinces located in the north (Hai Phong, Bac Ninh, Nghe An), northwest (Dien Bien, yen Bai) and northeast (Quan ninh) of the country. Evidence suggests that an overall low prevalence in recent times neither assures stability nor confirms absence of risk behaviors among IDUs as a whole [17], rather, it may shadow the growth of localized epidemics in many pockets 
especially in the high prevalent provinces [1].

Historically, 'shooting galleries', typically a common place for congregation of IDUs, can be found in clandestine locations that often provide opportunities for buying, renting, or borrowing needles/syringes and other injection items $[18,19]$. Injecting in shooting galleries and sharing common water pots have been found to be independent predictors for HIV infection among IDUs in Vietnam [20].

In addition to the above drug related risk practices, around $50 \%$ of IDUs in Vietnam visit Female Sex Workers (FSWs) $[11,21]$. Research shows that in different instances around $2-7 \%$ of IDUs either provided money or supplied drugs to FSWs (within the past 6 months) in exchange for sex [22]. Moreover, among the FSWs, a high proportion also takes drugs and, of concern, around $15-20 \%$ of them are engaged in injecting and sharing practices [23-28].

This background highlights an existing epidemic situation relating high risk behaviors of IDUs as well as intersection with FSWs, which together influence the current HIV prevalence among IDUs. The existing research literature, over time, describes the history of Vietnam's HIV epidemic showing the prevalence among different risk groups, but little is reported about the pattern and extent of risk behaviors among IDUs connecting with a risk of greater heterosexual transmission. It is important to review the available literature to explore findings on IDU risk behaviors, in order to focus on major issues which carry implications that might exacerbate the current epidemic. Once such issues are well identified, preventative efforts can be better targeted and service provision rendered effectively and efficiently. This paper aims to review the relevant published literature to analyze the risk behaviors among IDUs and identify the implications for the current epidemic.

\section{Methods}

This review commenced in August, 2011, as part of a broader research initiative. The research team includes members from the Vietnam Authority of HIV/AIDS Control (VAAC) and Griffith University (GU). GU library databases including PubMed, ProQuest, Science Direct were used to conduct the literature search during November-December, 2011. A combination of key-words such as 'HIV/AIDS in Vietnam', 'IDU and Vietnam', 'Drug Use and HIV Risk in Vietnam' along with the preferred time limit of '1995 onwards' were used to search the literature. Often the technical procedures of a systematic search such as strict selection criteria may mean that articles published with different titles are missed, thereby generating limited information. Such 'excluded' literature may provide crucial insights in the areas of interest. This is common in situations with limited availability of peer reviewed literature. Because of this factor, both a manual search and an exploration of relevant references were conducted to include a range of literature. After simultaneous searching, both peer-reviewed and non-peer reviewed documents were identified and reviewed. Peer reviewed articles were selected based upon reviewing risk behaviors among IDUs and mixing patterns with FSWs, whereas non-peer reviewed national and international documents provided contextual understanding of HIV, drug use problems and Harm Reduction (HR) programs in Vietnam.

Since the present review theme encompasses a range of related areas, the selected peer reviewed articles focus on (1) IDUs, (2) FSWs, and (3) drug and HR policies. After reviewing abstracts a total of 55 peer reviewed articles were identified, with 40 on IDU risk behaviors; 10 on FSWs to highlight patterns of sexual mixing among FSWs; and 5 on policy and HR program development. Furthermore, 10 non-peer reviewed documents were identified including surveillance results, country progress report and conference papers. These documents were used to contextualize the findings in terms of HR programs among IDUs in Vietnam. This review mainly concentrates on the 40 selected articles dealing with IDU HIV risk behaviors.

Of the 40 peer reviewed articles, 32 are based on original research and the remaining 8 deal with national level epidemiological data (surveillance, case reports). Out of the 32 research articles, 22 and 7 represent northern and southern Vietnam respectively, while 3 articles represent both. The majority (30) of the articles were published during second decade of the HIV epidemic (2001-2010) with only 10 published during the first five years (1995-2000). All the selected research articles deal with risk behaviors such as IDUs only (26) or combine IDUs, FSWs and other groups (9) or HIV infected IDUs (4), but only one represents risk behaviors among young people in general. The majority of the articles (38) are based on cross-sectional research designs, apart from one case-control and one cohort study. Most of the articles (30) present quantitative analyses and 6 present qualitative, while the remaining 4 use mixed methods. During the review process, articles which provided HIV prevalence data along with risk behaviors (nearly one-third) were prioritized.

\section{Results}

The review results relating HIV risk behaviors and other issues were classified into the following categories: (i) demographic, (ii) drug, (iii) sex, and (iv) overlapping drug and sex related issues.

\subsection{Demographic Issues}

The evidence indicates that IDUs in Vietnam are predominantly male $(80-90 \%)$ and the majority are single $(60-70 \%)$. The mean age, according to the literature after 2000 , shows a majority of IDUs below 30 years, ranging from 20-29 years.

The heroin shipment route which connects selected northern provinces with the 'Golden Triangle' creates an ample supply of heroin at a low cost and with easy access [29], thus facilitating the growth of the twin epidemics of heroin injection and HIV [30]. In Vietnam, drug users older than 20 years $(\mathrm{OR}=2.76 ; 95 \% \mathrm{CI}: 1.80-4.24)$ and using 
drugs for more than 5 years $(\mathrm{OR}=2.06 ; 95 \% \mathrm{CI}: 1.32-3.21)$ are predictors of the move to injectable drugs [11]. Consequently, injecting heroin has become the preferred drug among IDUs in Vietnam [31].

Moreover, the issue of speed of transition from non-injecting to injecting drug use among young IDUs is striking. Evidence shows that $57 \%$ of a sample of IDUs had switched to injecting practices and the average time for transition was just over one year (14 months) [12]. In other research the transition period was even less than one year [9, 32]. In Karachi, Pakistan, as a case study in recent modelling, it is projected that there will be a $50-65 \%$ rise in HIV infection over a period of 5 years, due to an $8-12 \%$ shift from non-injecting to injecting drug [33].

Injecting in places other than homes magnifies the level of risk behaviors in drug acquisition. There is a higher likelihood of needle sharing for IDUs who inject either at a shooting gallery $(\mathrm{OR}=11.94 ; 95 \% \mathrm{CI}: 4.07-34.99)$ or in the streets/ (public places) $(\mathrm{OR}=10.52 ; 95 \% \mathrm{CI}: 2.86-38.78)$ in comparison to those who inject at other places, such as private homes, or friend's houses [20]. Among IDUs who are already infected with HIV, the age range 25-29 years $(\mathrm{OR}=2.1 ; 95 \% \mathrm{CI}: 1.59-2.68)$ is found to be predictive for sharing needles and syringes in the previous month [16].

\subsection{Drug Use Issues}

Sharing needles/syringes and other injection equipment is the most frequent drug related risk behavior which puts IDUs at risk of virus transmission [34]. In Vietnam, needle/syringe sharing has been widespread in the shooting galleries which were operated by the drug dealers and research has confirmed that sharing in these places is a primary risk behavior [35, 36]. At the beginning of the epidemic, for example, more than $80 \%$ of IDUs shared syringes with unknown IDU participants at their last injecting occasion at a shooting gallery [35]. More than one-third (37\%) of IDUs had a life time practice of sharing of needles and syringes; however, the proportion drops to $14 \%$ when estimating sharing within the last six months [37]. As the epidemic progressed, being injected by a drug dealer, or someone else, became one of the predictors for HIV infection [20] and subsequently led to high risk sexual behaviors as well (OR=2.28; 95\%CI:1.16-4.51) [38].

Sharing as well as reusing of a previously used needle/syringe (direct sharing), over the period, has decreased among IDUs due to an improved level of risk awareness. A case-control research study among IDUs in Bac Ninh, a northern province, found that $13.3 \%$ of subjects had directly reused someone else's syringe at least once within the last six months [39]. In Thanh Hoa, 15\% IDUs reported sharing of needles/syringes during the last month [40]. The prevalence of other blood-borne viral infections supplies evidence indicating continued sharing and engagement with partners who belong to other risk groups [39, 41, 42]. Research confirms that IDUs who share needles are more likely to be infected with HIV than those who do not share needles [20,38].
Although there is evidence of decreased sharing of needles/syringes, there is a lack of research regarding indirect modes of transmission (indirect sharing) [43]. Evidence indicates that the majority of the subjects (63.8\%) shared indirectly, which includes using common drug containers, rinse water and cotton-wool, thus serving inadvertently as a transmission link [39]. In Lang Son, another northern province participating in a cross-border HIV prevention program, nearly one-third of the respondents in an evaluation reported sharing an injection item during the last 6 months [22]. IDUs who are involved in social injecting by pooling money in groups to buy drugs are often involved in indirect sharing behaviors [16, 41]. Alarmingly, people who are infected with HIV (PLHIV) appear to continue their drug related risk behaviors [44-46].

The sharing practice is deeply rooted within the IDU social and cultural context [47, 48]. In addition, other frequently cited reasons for sharing include limited resources, lack of clean needles/syringes and fears of arrest by law personnel, all of which reinforce reusing and sharing equipment $[12,44,46]$. One study identifies cross border factors as reasons for sharing among young ethnic IDUs in border areas (south China and north Vietnam) [22, 49]. About two-thirds (64\%) of the IDUs indicated that injecting at a shooting gallery was the primary reason for sharing needles [37].

\subsection{Sex Work Issues}

IDUs provide an effective epidemiologic bridge for the generalized epidemic through unsafe sexual practices with their regular partners (wives) and non-regular partners (FSWs) [23]. Around 58\% of male IDUs had more than 5 lifetime sex partners and more than 2 sex partners in the last 12 months [50]. Moreover, such sexual connection spans across drug use and sex work networks including casual partners [12] and often newer and younger IDU subgroups [11, 26]. Furthermore, sexual risk behaviors are prevalent among HIV sero-positive IDUs $[13,51]$ and often shows recent involvement, as in the previous week [45].

As a common belief, IDUs perceive the risk of virus transmission is less likely through sexual practice than sharing injection items [26] and hence they are less likely to adopt safe sex than safe injection practices [37, 48]. As a result, non-condom sex with FSWs is widespread $[52,53]$. Among the IDUs in HCM city who reported sex with FSWs within the last 4 weeks $(15 \%)$, only one-half $(50 \%)$ used condoms [37]. It is evident that around $15 \%$ of IDUs in Bac Ninh, a northern province, were inconsistent in their use of condoms and another $15 \%$ did not like to use them while having sex with FSWs [50]. In other instances, self-reported condom use among IDUs reached almost a quarter $(25 \%)$ in Hai Phong province when having sex with FSWs, but dropped below $10 \%$ when having sex with their regular partners [11]. Consequently, STI prevalence has increased among IDUs [50], and FSWs [54, 55] their clients [56] which, in turn, facilitates transmission of HIV infection [50]. As a result of continuous unprotected sexual 
behaviors, a gradual increase of HIV infection among pregnant women in selected provinces has already been recorded [57].

Relationship and communication dynamics between IDUs and their sex partners is important as it shapes their sexual behaviors [53, 58]. Although the primary female partners of male IDUs may wish to use condoms, they face major barriers such as lack of power, fearing to ask, fear of breaking trust and often partners not allowing them to use condoms [51, 52].

\subsection{Overlapping Drug Use and Sex Work Issues}

Injecting, together with unprotected sexual practices, is an already established combination among IDUs in Vietnam [8]. Sharing needles (OR=2.43; 95\%CI: 1.08-5.50) and sharing any injection equipment $(\mathrm{OR}=1.88 ; 95 \% \mathrm{CI}$ : 1.01-3.50) in the past six months is also associated with risky sexual practice over the past year [38]. For young IDUs, for instance, who sometimes shared needles (22.5\%), more than half $(55.6 \%)$ never used condoms during sex with their partners [13]. The situation with drug injecting young FSWs (less than 25 years) is even worse $(\mathrm{OR}=5.22$; 95\% CI: 2.10-12.97) and is independently associated with HIV sero-positivity in HCM city [28].

Consequently, injecting drug use is considered as a key issue affecting the rapid increase of HIV infection among FSWs [28, 59-61]. Having drug injecting partners $(\mathrm{OR}=10.0 ; 95 \% \mathrm{CI}: 5.2-20)$ and having drug using clients $(\mathrm{OR}=2.5$; 95\%CI: 1-6) are predictors for injecting drugs among FSWs [27]. FSWs who inject drugs have a 7.8 times higher chance of getting HIV infection than those who do not inject drugs [43]. In addition, the rise of HIV infection among FSWs is significantly associated with risky sexual behaviors (frequency of sex acts, inconsistent use of condoms, multiple clients) [55, 62, 63]. In summary, because of frequent overlapping risk behaviors, the evidence supports the emergence of two epidemics which may substantially inhibit opportunities for HIV prevention in the future [64].

\section{Discussion}

This review of HIV risk behaviors and related issues has found a high rate of sharing practices since the beginning of the epidemic that dropped gradually with improvement in risk awareness knowledge among IDUs. However, the sharing practices, especially indirect modes of sharing continue and there are visible IDU subgroups, especially younger IDUs (under 30 years of age), who frequently engage in HIV risk behaviors. Evidence suggests that infrequent contact with HR staff impedes the accessibility of sterile needles/syringes for such young injectors and thus fails to promote risk reduction [65]. Similarly, new injectors are also younger (less than 30 years of age) [66] and tend to be more drug-dependent together with increased risk behaviors [12, 26, 38]. This review identifies that young drug users facilitated the recent spread of HIV infection among IDUs and these factors must be addressed with an innovative HR project design approach.

Secondly, the chain of infection developed through drug and sex related contacts, indicates the development of an effective epidemiologic bridge to deliver HIV infection to sexual partners (SP) in non-high risk populations. Although the risk associated with SPs has been identified and included for prevention services on a limited scale [58], such coverage needs to be extended before the prevalence rises further. Epidemic models demonstrate a complex role of IDUs [67] and indicate that high rates of sexual behavior facilitate the risk of an injecting-driven HIV epidemic progressing towards a generalized epidemic [68, 69]. A strong link to exacerbate the current epidemic already exists $[43,70,71]$ with young and new injectors in Hanoi, who are sexually active, having the potential to spread the disease to their SPs, in general FSWs, of whom around $20 \%$ are already infected [2, 24, 26, 27]. Innovative sexual health programs should be implemented covering young FSWs and IDU subgroups, before the rate of mixing reaches or crosses the threshold level that facilitates the growth of further heterosexual transmission.

The third major finding relates to the overlapping risk behaviors of drug-using women, especially injecting FSWs, which is another group to target for prevention. Drug using male partners and clients influence the drug use behavior of FSWs, which possibly explains the concurrent spread of HIV infection among male IDUs as well as FSWs [8, 27, 43]. Female IDUs may become potential catalysts when heterosexual transmission becomes more prevalent. This is not denying that HIV infection among non-injecting male drug users may be a result of engagement in sexual relationship with drug-using female partners.

Finally, another finding is the engagement in risk behaviors of persons who are already infected with the virus, regardless of whether they are drug injecting males or FSWs. A possible reason could be the ineffective implementation of community-based HIV testing services which failed to achieve the benefits of in-depth counselling among PLHIV $[16,72]$. Therefore, the need for scaling up of HIV counselling among IDUs with improved care and quality services is also required [73].

This review supports the contention that the implementation of HR programs among IDUs in Vietnam has not been uniform [74] and this may account for continuing risk behaviors in different parts of the country. Recent change in policies (replacing the 'social-evil' approach with 'health-rights' paradigm) has broadened the scope of extensive HR programs, including subgroup members [75, 76]. Increased and easy access to sterile injection equipment helps to motivate young and new drug users to accept safer behaviors [40, 77]. Historical experience [78] as well as lessons from neighboring countries, such as Cambodia and Thailand, suggests that targeted interventions with high coverage is effective to slowdown the epidemic [79].

There are some limitations also which needs to be 
reported. The review looks into HIV prevalence and risk behaviors only, while other issues related to HR programs and drug policies have not been analyzed in detail. Similarly, documents showing major risk behaviors among IDUs were prioritized, whereas the research articles concerning FSWs which were not selected (around 20) may contain risk behaviors among FSWs and other clients and may have reported implications for a future expanded epidemic. Another limitation of this review is that it includes peer reviewed articles published in English, accompanied by other national and international non-peer reviewed documents. Consensus has made among research team members regarding identified English language literature to focus and analyze risk behaviors. Despite such limitations, however, the findings above are crucial and show a heightened need for HR program to prevent the further spread of the HIV infection.

Future research should primarily focus on IDUs who are under 30 years of age and new users who have been injecting for 3-5 years, to investigate network structure and characteristics. In addition, research should focus on the structural factors associated with rapid transition. Other potential areas of research include sexual risk analysis associated with multiple partners and disease transmission from risk groups both to the SPs (in general) and target clients [80]. Furthermore, research should assess the potential for a clinic based dual screening of STI and HIV to maximize HIV testing uptake among women in general [81] especially in high prevalent provinces [57].

\section{Conclusion}

Young injectors who have sexual links with FSWs in major cities highlight the most critical prevention need at this stage of the epidemic in order to avoid future heterosexual transmission in Vietnam. This sexual mixing of IDUs heightens the risk of heterosexual transmission which could further worsen the epidemic situation. Vietnam still has a window of opportunity to observe the experiences of neighboring countries and incorporate lessons into their prevention programs [82]. The major challenge lies in reaching the younger, newer subgroups of IDUs with innovative interventions tailored to their service need.

\section{Acknowledgements}

The first author is a PhD candidate in Griffith University, Queensland, Australia and acknowledges the financial support of Australia Awards. He also acknowledges the assistance of Vietnam Authority of HIV/AIDS Control.

\section{References}

[1] Socialist Republic of Vietnam, The Fourth Country Report on Following-up the Implementation to the Declaration of Commitment on HIV and AIDS Vietnam Authority of HIV/AIDS Control: Hanoi. 2010.
[2] Ministry of Health, Results from the HIV/STI Integrated Biological and Behavioral Surveillance (IBBS) in Vietnam 2005-2006, 2006: Vietnam.

[3] Tran, H.N., et al., HIV monitoring in Vietnam: System, methodology, and results of sentinel surveillance. Journal of Acquired Immune Deficiency Syndromes, 1999; 4(21): 338.

[4] Chung, A., M.Q. Vu, and T.J. Dondero, HIV epidemiologic situation in Vietnam: A review of available data. AIDS, 1998. 12 Supplement B: S43-9.

[5] Lindan, C.P., et al., Rising HIV infection rates in Ho Chi Minh City Herald emerging AIDS epidemic in Vietnam. AIDS, 1997; 11 Supplement 1(0269-9370): S5-13.

[6] Hien, N.T., N.T. Long, and T.Q. Huan, HIV/AIDS epidemics in Vietnam: Evolution and responses. AIDS Education and Prevention, 2004; 16(Supplement A): 137-154.

[7] Nguyen, T.H. and I. Wolffers, HIV infection in Vietnam. The Lancet, 1994; 343(8894): 410.

[8] Quan, V.M., et al., HIV in Vietnam: The evolving epidemic and the prevention response, 1996 through 1999. Journal of Acquired Immune Deficiency Syndromes, 2000; 25(4): 360-369.

[9] Tran, S.D., et al., Risk factors for HIV sero-positivity in a sample of drug users in drug treatment in Ho Chi Minh City, Vietnam. Journal of Acquired Immune Deficiency Syndrome and Human Retrovirology, 1998; 17(3): 283-287.

[10] Follézou, J.Y., et al., Clinical and biological characteristics of human immunodeficiency virus-infected and uninfected intravascular drug users in Ho Chi Minh City, Vietnam. The American Journal of Tropical Medicine and Hygiene, 1999; 61(3): 420-4.

[11] Nguyen, T.A., et al., Risk factors for HIV-1 seropositivity in drug users under 30 years old in Haiphong, Vietnam. Addiction, 2001; 96(3): 405.

[12] Thao, L., et al., Changes in high-risk behaviors over time among young drug users in south Vietnam: A three-province study. AIDS and Behavior, 2006; 10(0): 47-56.

[13] Tran, T.M.T., et al., HIV prevalence and factors associated with HIV infection among male injection drug users under 30: A cross-sectional study in Long An, Vietnam. BMC Public Health, 2006; 6: 248.

[14] Hammett, T.M., et al., Correlates of HIV status among injection drug users in a border region of southern China and northern Vietnam. Journal of Acquired Immune Deficiency Syndromes, 2005; 38(2): 228-235.

[15] Ministry of Health, Results from the HIV/STI Integrated Biological and Behavioral Surveillance (IBBS) in Vietnam (Round I I) 2000 , National Institute of Hygiene and Epidemiology and Family Health International: Hanoi, Vietnam.

[16] Go, V.F., et al., Characteristics of high-risk HIV-positive IDUs in Vietnam: Implications for future interventions. Substance Use and Misuse, 2011; 46(4): 381-389.

[17] Des Jarlais, D.C., et al., HIV-1 infection among intravenous drug users in Manhattan, New York city, from 1977 through 1987. The Journal of the American Medical Association, 1989; 261(7): 1008-1012. 
[18] Des Jarlais, D.C., H. Hagan, and S.R. Friedman, Drug Abuse and Spread of Infection: HIV and AIDS as an Example, In: Epidemiology of Drug Abuse, Z. Sloboda, Editor. Springer; 2005. p. 193-208.

[19] Celentano, D.D., et al., Risk factors for shooting gallery use and cessation among intravenous drug users. American Journal of Public Health, 1991; 81(10): 1291-1295.

[20] Hien, N.T., et al., Risk factors of HIV infection and needle sharing among injecting drug users in Ho Chi Minh City, Vietnam. Journal of Substance Abuse, 2001; 13(1-2): 45 - 58.

[21] Family Health International and National AIDS Standing Bureau, HIV/AIDS Behavioral Surveillance Survey Vietnam 2000, 2001: Hanoi.

[22] Hammett, T.M., et al., Patterns of HIV prevalence and HIV risk behaviors among injection drug users prior to and 24 months following implementation of cross-border HIV prevention interventions in northern Vietnam and southern China. AIDS Education Prevention, 2006; 18(2): 97-115.

[23] Ministry of Health and Population Reference Bureau, HIV/AIDS in Vietnam: The Current Situation, the National Response, the Emerging Challenges, 2007: Hanoi/Washington, DC. .

[24] Monitoring AIDS Pandemic, MAP Report 2005 Drug Injection and HIV/AIDS in Asia, 2005, The Monitoring AIDS Pandemic Network. p. 19.

[25] Tran, T.N., et al., Drug use, sexual behaviours and practices among female sex workers in Hanoi, Viet Nam-A qualitative study. International Journal of Drug Policy, 2004; 15(3): 189-195

[26] Tran, T.N., et al., Drug use, sexual behaviours and practices among male drug users in Hanoi, Vietnam-A qualitative study. International Journal of Drug Policy, 2004; 15(3): 182-188.

[27] Tran, T.N., et al., Drug use among female sex workers in Hanoi, Vietnam. Addiction, 2005; 100: 619-625.

[28] Tuan, N.A., et al., Intravenous drug use among street-based sex workers: A high-risk behavior for HIV transmission. Sexually Transmitted Diseases, 2004; 31(1): 15-19.

[29] Beyrer, C., et al., Overland heroin trafficking routes and HIV-1 spread in south and south-east Asia. AIDS, 2000; 14(1): $75-83$

[30] Maher, L., Entwined epidemics: HIV and injecting drug use in Vietnam. International Journal of Drug Policy, 2004; 15(3): 169-170.

[31] Cohen, J., HIV and Heroin: A Deadly International Affair. Science, 2003; 301(5640): 1657-1658.

[32] Nguyen, T., et al., Explosive HIV epidemic among young heroin injecting users in Quang ninh province, Vietnam: Risk factors for HIV seropositivity, in International Conference on AIDS. 2002; A.N. MoPeC3387: 2002.

[33] Strathdee, S.A., et al., HIV and risk environment for injecting drug users: the past, present, and future. The Lancet, 2010; 376(9737): 268-284.

[34] Institute of Medicine, Preventing HIV Infection among Injecting Drug Users in High-Risk Countries: An Assessment of the Evidence. 2006, Washington DC: National Academic Press.
[35] Power, R., Rapid assessment of the drug-injecting situation at Hanoi and Ho Chi Minh City, Viet Nam. Bulletin of Narcotics, 1996; 48(1-2): 35-52.

[36] Abdul-Quader, A.S., V.M. Quan, and K. O'Reilly, A tale of two cities: HIV risk behaviours among injecting drug users in Hanoi and Ho Chi Minh City, Vietnam. Drug and Alcohol Review, 1999; 18(4): 401-407.

[37] Hien, N.T., et al., The social context of HIV risk behaviour by drug injectors in Ho Chi Minh City, Vietnam. AIDS Care, 2000; 12(4): 483-495.

[38] Schumacher, C.M., et al., Social injecting and other correlates of high-risk sexual activity among injecting drug users in northern Vietnam. International Journal of Drug Policy, 2009; 20(4): 352-356.

[39] Quan, V.M., et al., Risks for HIV, HBV, and HCV infections among male injection drug users in northern Vietnam: a case-control study. AIDS Care, 2009; 21(1): 7-16.

[40] Son, V.H. Risk behavior among Injecting Drug Users in Thanh Hoa province, Vietnam in3rdInternational Conference on Reproductive Health and Social Sciences Research 2009. Royal City Hotel, Bangkok, Thailand: Institute for Population and Social Research (IPSR), Mahidol University.

[41] Clatts, M.C., et al., Prevalence and incidence of HCV infection among Vietnam heroin users with recent onset of injection. Journal of Urban Health, 2010; 87(2): 278-91.

[42] Nguyen, C.H., et al., Prevalence of HBV infection among different HIV-risk groups in Hai Phong, Vietnam. Journal of Medical Virology, 2011; 83(3): 399-404.

[43] Tuan, N.A., et al., Human immunodeficiency virus (HIV) infection patterns and risk behaviours in different population groups and provinces in Viet Nam. Bulletin of the World Health Organization, 2007; 85(1): 35-41.

[44] Khoat, D.V., et al., Peer education for HIV prevention in the socialist republic of Vietnam: A national assessment. Journal of Community Health, 2003; 28(1): 1-17.

[45] Thanh, D.C., et al., HIV risk behaviours and determinants among people living with HIV/AIDS in Vietnam. AIDS and Behavior, 2009; 13(6): 1151-9.

[46] Thanh, D., K. Moland, and K. Fylkesnes, The context of HIV risk behaviours among HIV-positive injection drug users in Viet Nam: Moving toward effective harm reduction. BMC Public Health, 2009; 9(1): 98.

[47] Howard, J. and P. Borges, Needle sharing in the Haight: Some social and psychological functions. Journal of Health and Social Behavior, 1970; 11(3): 220-230.

[48] Lam, N.T., Drugs, sex and AIDS: Sexual relationships among injecting drug users and their sexual partners in Vietnam. Culture, Health \& Sexuality, 2008; 10(sup1): S123-S137.

[49] Des Jarlais, D.C., et al., Patterns of HIV prevalence among injecting drug users in the cross-border area of Lang Son province, Vietnam, and Ning Ming county, Guangxi Province, China. BMC Public Health, 2005; 5: 89.

[50] Go, V.F., et al., High HIV sexual risk behaviors and sexually transmitted disease prevalence among injection drug users in northern Vietnam: Implications for a generalized HIV epidemic. Journal of Acquired Immune Deficiency Syndromes, 2006; 42(1): 108-115 
[51] Rosenthal, D. and T.T.K. Oanh, Listening to female sex workers in Vietnam: influences on safe-sex practices with clients and partners. Sexual Health, 2006; 3(1): 21-32.

[52] Detels, R., Condom use and its correlates among female sex workers in Hanoi, Vietnam. AIDS and Behavior, 2006; 10(2): $159-67$.

[53] Go, V.F., et al., Intra-couple communication dynamics of HIV risk behavior among injecting drug users and their sexual partners in northern Vietnam. Drug and Alcohol Dependence, 2006; 84(1): 69-76.

[54] Grayman, J.H., et al., Factors associated with HIV testing, condom use, and sexually transmitted infections among female sex workers in Nha Trang, Vietnam. AIDS and Behavior, 2005; 9(1): 41-51.

[55] Mai, T.T.T., L.C. Linh, and N.T. Long, Factors associated with inconsistent condom use among female sex workers in Nha Trang, Vietnam. Asia-Pacific Journal of Public Health, 2008; 20(4): 370-378.

[56] Lindan, C.P., et al., Predictors of visits to commercial sex workers by male attendees at sexually transmitted disease clinics in southern Vietnam. AIDS, 1999; 13(6): 719-725.

[57] Quan, V.M., N.T. Hien, and V.F. Go, The HIV Epidemic in Vietnam: Past, Present, and Opportunities in Public Health Aspects of HIV/AIDS in Low and Middle Income Countries: Epidemiology, Prevention and Care, D. Celentano and C. Beyrer, Editors. Springer; 2009: p. 457-479.

[58] Hammett, T.M., et al., Female sexual partners of injection drug users in Vietnam: An at-risk population in urgent need of HIV prevention services. AIDS Care, 2010; 22(12): 1466-1472.

[59] Detels, R. and H.T. Long, HIV infection and risk characteristics among female sex workers in Hanoi, Vietnam. Journal of Acquired Immune Deficiency Syndromes, 2005; 39(5): 581-586.

[60] Nemoto, T., et al., HIV-related risk behaviours among female sex workers in Ho Chi Minh City, Vietnam. AIDS Education and Prevention, 2008; 20(5): 435-53.

[61] $\mathrm{Vu}, \mathrm{L}$. and C. Lindan, Evaluation of STD/HIV prevention needs of low- and middle-income female sex workers in Ho Chi Minh city, Vietnam. AIDS and Behavior, 2000; 4(1): 83.

[62] Thuy, N.T.T., et al., HIV infection and risk factors among female sex workers in southern Vietnam. AIDS, 1998; 12(4): 425-432.

[63] Thuong, N.V., et al., HIV in female sex workers in five border provinces of Vietnam. Sexually Transmitted Infections, 2005; 81(6): 477-479.

[64] Ghys, P.D., et al., Growing in silence: Selected regions and countries with expanding HIV/AIDS epidemics. AIDS, 2003; 17 Supplement 4(0269-9370): S45-S50.

[65] Bailey, S.L., et al., The use of needle exchange by young injection drug users. Journal of Acquired Immune Deficiency Syndromes, 2003; 34(1): 67-70.

[66] Vu, T., Harm Reduction for Injecting Drug User in Vietnam: A situation Assessment, in Report for Macfarlane Burnet centre. Victorian Public Health Training Scheme2001: Melbourne.
[67] Wiessing, L. and M. Kretzschmar, Can HIV epidemics among IDUs 'trigger' a generalised epidemic? International Journal of Drug Policy, 2003; 14(1): 99-102.

[68] Grassly, N.C., et al., Modelling emerging HIV epidemics: The role of injecting drug use and sexual transmission in the Russian Federation, China and India. International Journal of Drug Policy, 2003; 14(1): 25-43.

[69] Saidel, T.J., et al., Potential impact of HIV among IDUs on heterosexual transmission in Asian settings: scenarios from the Asian Epidemic Model. International Journal of Drug Policy, 2003; 14(1): 63-74.

[70] Duong, C.T., et al., Sexual risk and bridging behaviors among young people in Hai Phong, Vietnam. AIDS and Behavior, 2008; 12(4): 643-51.

[71] Truong, T.M., G.R. West, and T.M. Durant, Sex workers in Vietnam: How many, how risky? AIDS Education and Prevention, 2004; 16(5): 389-404.

[72] Bergenstrom, A., et al., Return to post-test counselling by out-of-treatment injecting drug users participating in a cross-sectional survey in north Vietnam. AIDS Care, 2007; 19(7): 935-939.

[73] Maher, L., H. Coupland, and R. Musson, Scaling up HIV treatment, care and support for injecting drug users in Vietnam. International Journal of Drug Policy, 2007; 18(4): 296-305.

[74] Reid, G. and P. Higgs, Vietnam moves forward with harm reduction: an assessment of progress. Global Public Health, 2011; 6(2): 168-180.

[75] Ha, P.N., et al., The evolution of HIV policy in Vietnam: From punitive control measures to a more rights-based approach. Global Health Action, 2010; 3(1654-9880).

[76] Hammett, T.M., et al., 'Social evils' and harm reduction: The evolving policy environment for human immunodeficiency virus prevention among injection drug users in China and Vietnam. Addiction, 2008; 103(1): 137-145.

[77] Des Jarlais, D.C., et al., Reducing HIV infection among new injecting drug users in the China-Vietnam Cross Border Project. AIDS, 2007; 21(0269-9370): S109-S114.

[78] Des Jarlais, D.C., et al., Maintaining low HIV seroprevalence in populations of injecting drug users. The Journal of the American Medical Association, 1995; 274(15): 1226-1231.

[79] Detels, R., HIV/AIDS in Asia: Introduction. AIDS Education and Prevention, 2004; 16(08999546): 1-6.

[80] Des Jarlais, D.C. and S. Semaan, Interventions to reduce the sexual risk behaviour of injecting drug users. International Journal of Drug Policy, 2005; 16, Supplement 1(0): 58-66.

[81] Tucker, J.D., et al., A twin response to twin epidemics: Integrated HIV/Syphilis testing at STI clinics in south China. Journal of Acquired Immune Deficiency Syndromes, 2011; 57(5): e106-e111.

[82] Gorbach, P.M., et al., The impact of social, economic and political forces on emerging HIV epidemics. AIDS, 2002; 16: S35-S43. 